ten mit einer nicht-systemischen JIA: 1047 wurden erstmals auf Etanercept, 304 auf Adalimumab ( $n=243) /$ Infliximab $(n=61)$ und 943 (Kontrollkollektiv) auf Methotrexat eingestellt. Eine vorangegangene Uveitis stellte ein Ausschlusskriterium dar. Anhand der medizinischen Dokumentationen objektivierten die Forscher, wie viele Patienten im Therapieverlauf eine Uveitis entwickelten. Die Nachbeobachtungszeit begann mit der ersten Behandlung und endete mit der ersten Uveitisdiagnose, dem Absetzen/Wechsel des Medikaments, dem jüngsten Betreuungstermin bzw. dem Tod.

\title{
Ergebnisse
}

Im Verlauf von 5456 Personenjahren Nachbeobachtungszeit erlitten 44 Studienpatienten eine Uveitis, darunter einer der mit Adalimumab behandelten Patienten, $16 \mathrm{~Pa}$ tienten der Etanercept-Gruppe sowie 27

\section{Juvenile idiopathische Arthritis: Uveitisrisiko unter verschiedenen} \section{TNF-Inhibitoren}

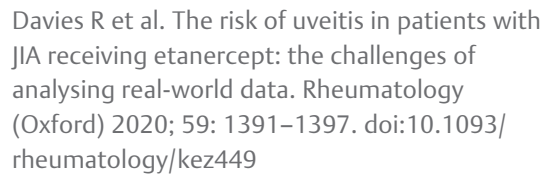

Ein erheblicher Teil der Patienten mit einer juvenilen idiopathischen Arthritis (JIA) entwickelt im Krankheitsverlauf eine Uveitis. Inwiefern beeinflusst die Behandlung mit den TNF-Inhibitoren Etanercept, Adalimumab und Infliximab das Neuerkrankungsrisiko? Mit dieser Fragestellung beschäftigte sich ein Team britischer Forscher.

Die Wissenschaftler werteten die Daten zweier britischer prospektiver JIA-Register aus: Der „British Society for Paediatric and Adolescent Rheumatology Etanercept Cohort Study“ (BSPAR-ETN) und des „Biologics for Children with Rheumatic Diseases“ (BCRS). Die Analyse umfasste 2294 Patien- 
Kontrollen. In der Mehrzahl der Fälle handelte es sich dabei um eine einseitige Problematik und meist war das anteriore Kompartiment betroffen. Eine Panuveitis trat im Studienkollektiv nicht auf. Das Durchschnittsalter der Kinder bei der Uveitisdiagnose betrug in der Etanercept-Gruppe 7 und in der Methotrexat-Gruppe 4 Jahre. Seit der JIA-Diagnose waren in diesen beiden Kohorten 4 bzw. 2 Jahre vergangen. Der unter Adalimumab erkrankte Patient war älter als 15 Jahre und litt seit 2 Jahren an der JIA. In der nicht adjustierten Analyse deutete sich zunächst im Kollektiv der mit Biologika behandelten Patienten im Vergleich zu Methotrexat ein reduziertes Uveitisrisiko an. Der bezüglich Propensity-Dezilen adjustierte Vergleich Etanercept-Methotrexat bestätigte dies jedoch nicht. Ein vollständig adjustierter Vergleich Adalimumab/Infliximab-Methotrexat war angesichts geringer Ereigniszahlen nicht möglich.
FAZIT

Mit einem TNF-Inhibitor behandelte JIA-Patienten erkranken im Vergleich zu mit Methotrexat behandelten Kindern tendenziell seltener an einer Uveitis - statistische Signifikanz besteht diesbezüglich allerdings nicht, so die Autoren. Sie führen den vermeintlich protektiven EtanerceptEffekt auf Störvariablen zurück: Die Methotrexat-Kinder hatten angesichts ihres durchschnittlich jüngeren Alters sowie der kürzeren JIA-Dauer im Vergleich zu den EtanerceptPatienten per se ein höheres Uveitisrisiko.

Dr. med. Judith Lorenz, Künzell 